\title{
Applications of quadrivariate exponential distribution to a three-unit warm standby system with dependent structure
}

\author{
V.S.S. Yadavallia, ${ }^{*}$, V.S. Vaidyanathan ${ }^{b}$, P. Chandrasekhar ${ }^{c}$, and S. Abbas ${ }^{a}$ \\ aDepartment of Industrial and Systems Engineering, University of Pretoria, Pretoria, South Africa; \\ bDepartment of Statistics, Pondicherry University, Puducherry, India; \\ 'Department of Statistics, Loyola College, Chennai, Tamil Nadu, India \\ *CONTACT: V. S. S. Yadavalli, Department of Industrial \& Systems Engineering, University of Pretoria, \\ Pretoria, 2000, South Africa. Email: sarma.yadavalli@up.ac.za
}

\begin{abstract}
Two-unit warm standby systems have been elaborately dealt within the literature. However, the study of standby systems with more than two units, though very relevant in state-of-the-art practical situations, has received little attention because of mathematical intricacies involved in analyzing them. Also, such systems have been studied assuming: (i) the lifetime or repair time of the units to be exponential, or (ii) the life-time and repair time to be independent. The present contribution is an improvement in the state-of-the-art in the sense that three-unit warm standby system with dependent structure is shown to be capable of comprehensive analysis.
\end{abstract}

KEYWORDS

Multivariate Central Limit theorem; Slutsky theorem; Standby system; Steady state availability.

\section{Introduction}

The purpose of this work is to study the system reliability, availability, and to obtain associated statistical inference for a three-unit warm standby system with dependent structure. The present work is an extension of the results obtained by Chandrasekhar et al. (2013) for a two-unit warm standby system with dependent structure. As pointed out by Srinivasan and Subramanian (2006), the study of three-unit warm standby systems is challenging because of the built-in intricacies involved in their analysis. Several authors have extensively studied two-unit standby redundant systems in the past. Osaki and Nakagawa (1976) gave a bibliography of the work on two-unit systems. Most of the studies on two-unit warm standby systems are confined to obtaining expressions for various measures of system performance and do not consider the associated inference problems. Chandrasekhar and Natarajan (1994) have considered a two-unit cold standby system and obtained the exact confidence limits for the steady state availability of the system under the assumption that the lifetime of online unit and the repair time of a failed unit are independent.

In general, the failure time and repair time need not be independent always. A system or a component that fails frequently within a short time interval has to be analyzed thoroughly, and the time taken to repair such a system will be more. The dependency between lifetime/failure time and repair time can be modeled by assuming a suitable form of a bivariate density function (see Figure 1). In the past, a number of bivariate exponential distributions have been proposed and studied well in the literature. But the bivariate exponential distribution of 


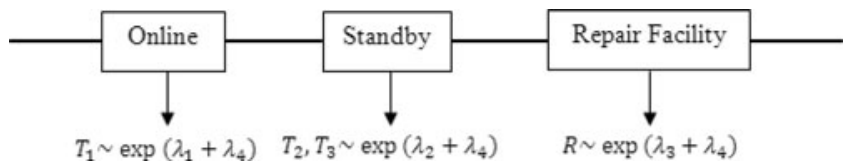

Figure 1. System configuration: three-unit warm standby system with repair.

Marshall and Olkin (1967) is widely accepted among many bivariate exponential distributions proposed in the statistical literature because of its nice properties. The present work is an attempt to analyze a three-unit warm standby system under the assumption that the joint distribution of the lifetimes of online unit, standby units, and the repair time of a failed unit in the system is quadrivariate exponential. Further, it is assumed that the lifetimes of the units kept in standby are identical. The model and the assumptions, expressions for reliability, availability, and associated statistical inference together with numerical illustration are discussed in detail in the following sections.

\section{Model (three-unit warm standby system)}

\subsection{Model and assumptions}

The system under consideration is a three-unit warm standby system with a single repair facility.

Precisely the following are the assumptions.

(i) The units are similar and statistically not independent. One unit is operating online and other two units are kept as warm standby. The three units have constant failure rates say $\left(\lambda_{1}+\lambda_{4}\right)$ while online and $\left(\lambda_{2}+\lambda_{4}\right)$ while in standby. Further, each failed unit has a constant repair rate say $\left(\lambda_{3}+\lambda_{4}\right)$.

(ii) There is only one repair facility.

(iii) Let $T_{1}, T_{2}$, and $T_{3}$ denote the lifetimes of the three units and $R$ the repair time of a failed unit in the system. As the system consists of three units with single repair, it is appropriate to consider the following Marshall-Olkin quadrivariate exponential (QVE) distribution for $T_{1}, T_{2}, T_{3}$, and $R$ with the survival function given by

$$
\begin{aligned}
& \bar{F}\left(t_{1}, t_{2}, t_{3}, t_{4}\right)=e^{-\left[\lambda_{1} t_{1}+\sum_{i=2}^{3} \lambda_{2} t_{i}+\lambda_{3} t_{4}+\lambda_{4} \cdot \max \left(t_{1}, t_{2}, t_{3}, t_{4}\right)\right]} \\
& t_{i}>0, i=1,2,3,4 ; \lambda_{i}>0, i=1,2,3 ; \lambda_{4} \geq 0
\end{aligned}
$$

and is denoted by $\left(T_{1}, T_{2}, T_{3}, R\right) \sim \operatorname{QVE}\left(\lambda_{1}, \lambda_{2}, \lambda_{2}, \lambda_{3}, \lambda_{4}\right)$ (see Marshall and Olkin, 1967).

(iv) Each unit is new after repair.

(v) Switch is perfect and the switchover is instantaneous.

Note:

1. The lifetimes of units $T_{1}, T_{2}$, and $T_{3}$ are exponential random variables each with the parameters $\left(\lambda_{1}+\lambda_{4}\right),\left(\lambda_{2}+\lambda_{4}\right)$, and $\left(\lambda_{2}+\lambda_{4}\right)$, respectively.

2. The repair time $R$ is exponential with the parameter $\left(\lambda_{3}+\lambda_{4}\right)$.

3. $\mathrm{E}\left(T_{1}\right)=\frac{1}{\left(\lambda_{1}+\lambda_{4}\right)} ; \mathrm{E}\left(T_{i}\right)=\frac{1}{\left(\lambda_{2}+\lambda_{4}\right)}, i=2,3$ and $\mathrm{E}(\mathrm{R})=\frac{1}{\left(\lambda_{3}+\lambda_{4}\right)}$; $\operatorname{Var}\left(T_{1}\right)=\frac{1}{\left(\lambda_{1}+\lambda_{4}\right)^{2}} ; \operatorname{Var}\left(T_{i}\right)=\frac{1}{\left(\lambda_{2}+\lambda_{4}\right)^{2}}, i=2,3$ and $\operatorname{Var}(\mathrm{R})=\frac{1}{\left(\lambda_{3}+\lambda_{4}\right)^{2}}$. 
4. The joint distribution of $\left(T_{1}, T_{j}\right), j=2,3$ is bivariate exponential (BVE) with the parameters $\left(\lambda_{2}, \lambda_{2}, \lambda_{4}\right)$ and joint distribution of $\left(T_{2}, T_{3}\right)$ is BVE with the parameters $\left(\lambda_{2}, \lambda_{2}, \lambda_{4}\right)$. Similarly, the joint distribution of $\left(T_{1}, R\right)$ is BVE with the parameters $\left(\lambda_{1}, \lambda_{3}, \lambda_{4}\right)$ and that of $\left(T_{i}, R\right), i=2,3$ is also BVE with the parameters $\left(\lambda_{2}, \lambda_{3}, \lambda_{4}\right)$.

5. The covariance between $T_{1}$ and $T_{j}$ is given by

$$
\operatorname{Cov}\left(T_{1}, T_{j}\right)=\frac{\lambda_{4}}{\left(\lambda_{1}+\lambda_{4}\right)\left(\lambda_{2}+\lambda_{4}\right)\left(\lambda_{1}+\lambda_{2}+\lambda_{4}\right)}, j=2,3
$$

Similarly, the following results can be established.

$$
\begin{aligned}
\operatorname{Cov}\left(T_{2}, T_{3}\right) & =\frac{\lambda_{4}}{\left(\lambda_{2}+\lambda_{4}\right)^{2}\left(2 \lambda_{2}+\lambda_{4}\right)} \\
\operatorname{Cov}\left(T_{1}, R\right) & =\frac{\lambda_{4}}{\left(\lambda_{1}+\lambda_{4}\right)\left(\lambda_{3}+\lambda_{4}\right)\left(\lambda_{1}+\lambda_{3}+\lambda_{4}\right)} \\
\operatorname{Cov}\left(T_{i}, R\right) & =\frac{\lambda_{4}}{\left(\lambda_{2}+\lambda_{4}\right)\left(\lambda_{3}+\lambda_{4}\right)\left(\lambda_{2}+\lambda_{3}+\lambda_{4}\right)}, i=2,3 .
\end{aligned}
$$

6. The lifetimes $T_{1}, T_{2}$, and $T_{3}$ and the repair time $R$ are independent if and only if $\lambda_{4}=0$.

7. If $\left(X_{i}, Y_{i}\right), i=1,2, \ldots, n$ is a random sample of size from a BVE population with parameters $\left(\lambda_{1}, \lambda_{2}, \lambda_{3}\right)$, then covariance between the sample means $\bar{X}$ and $\bar{Y}$ is given by $\operatorname{Cov}(\bar{X}, \bar{Y})=\frac{\lambda_{3}}{n\left(\lambda_{1}+\lambda_{3}\right)\left(\lambda_{2}+\lambda_{3}\right)\left(\lambda_{1}+\lambda_{2}+\lambda_{3}\right)}$.

8. The joint distribution of $\left(T_{1}, T_{2}, T_{3}\right)$ is trivariate exponential (TVE) with the parameters $\left(\lambda_{1}, \lambda_{2}, \lambda_{3}, \lambda_{4}\right)$. Further, the joint distributions of $\left(T_{1}, T_{j}, R\right), j=2,3$ and $\left(T_{2}, T_{3}, R\right)$ are (TVE) with the parameters $\left(\lambda_{1}, \lambda_{2}, \lambda_{3}, \lambda_{4}\right)$ and $\left(\lambda_{2}, \lambda_{2}, \lambda_{3}, \lambda_{4}\right)$, respectively.

\subsection{Analysis of system}

To analyze the behavior of the system, define $X(t)$ as the number of failed units at time $t$. The stochastic process $\{X(t), t \geq 0\}$ with the state space given by $E=\{0,1,2,3\}$ denotes the state of the system at time $t$. Since quadrivariate exponential distribution has exponential marginals and satisfies lack of memory property, it follows that the stochastic process describing the behavior of the system is a Markov process with infinitesimal generator $Q$ given by

$$
\begin{array}{cccc}
0 & 1 & 2 & 3 \\
1 \\
2 \\
3
\end{array}\left(\begin{array}{cccc}
-\left(\lambda_{1}+2 \lambda_{2}+3 \lambda_{4}\right) & \left(\lambda_{1}+2 \lambda_{2}+3 \lambda_{4}\right) & 0 & 0 \\
\left(\lambda_{3}+\lambda_{4}\right) & -\left(\lambda_{1}+\lambda_{2}+\lambda_{3}+3 \lambda_{4}\right) & \left(\lambda_{1}+\lambda_{2}+2 \lambda_{4}\right) & 0 \\
0 & \left(\lambda_{3}+\lambda_{4}\right) & -\left(\lambda_{1}+\lambda_{3}+2 \lambda_{4}\right) & \left(\lambda_{1}+\lambda_{4}\right) \\
0 & 0 & \left(\lambda_{3}+\lambda_{4}\right) & -\left(\lambda_{3}+\lambda_{4}\right)
\end{array}\right)
$$

It may be noted that the system upstates are $0,1,2$, while state 3 is the system downstate. Let $p_{i}(t)=\operatorname{Pr}[X(t)=i] \forall i \in E$ represent the probability that the system is in state $i$ at time $t$ with the initial condition $p_{0}(0)=1$. We assume that initially all the three units are operable and obtain the measures of system performance as follows: 


\subsubsection{System reliability}

The system reliability $R(t)$ is the probability of failure-free operation of the system in $(0, t]$. To derive an expression for the reliability of the system, we restrict the transitions of the Markov process to the upstates, namely 0,1 , and 2 . Using the infinitesimal generator of the process given in Equation (2), pertaining to these upstates, we derive the following differentialdifference equations:

$$
\begin{aligned}
& \frac{d p_{0}(t)}{d t}=-(a+2 b) p_{0}(t)+c p_{1}(t) \\
& \frac{d p_{1}(t)}{d t}=(a+2 b) p_{0}(t)-(a+b+c) p_{1}(t)+c p_{2}(t) \\
& \frac{d p_{2}(t)}{d t}=(a+b) p_{1}(t)-(a+c) p_{2}(t)
\end{aligned}
$$

where $a=\left(\lambda_{1}+\lambda_{4}\right), b=\left(\lambda_{2}+\lambda_{4}\right)$, and $c=\left(\lambda_{3}+\lambda_{4}\right)$.

Let $L_{i}(s)$ be the Laplace transform of $p_{i}(t), i=0,1,2$. Taking Laplace transforms on both the sides of the differential-difference equations given in Equations (3)-(5), solving for $L_{i}(s), i=0,1,2$, and inverting, we get $p_{0}(t), p_{1}(t)$, and $p_{2}(t)$. Thus, the system reliability is given by

$$
\begin{aligned}
& R(t)=\sum_{i=1}^{3} \\
& \quad \times \frac{\left\{\left[\alpha_{i}^{2}+(2 a+b+2 c) \alpha_{i}+\left(a^{2}+a b+a c+c^{2}\right)\right]+\left[(a+2 b)\left(\alpha_{i}+a+c\right)\right]+[(a+b)(a+2 b)]\right\}}{\prod_{j=1, j \neq i}^{3}\left(\alpha_{i}-\alpha_{j}\right)} e^{\alpha_{i} t}
\end{aligned}
$$

where $\alpha_{1}, \alpha_{2}$, and $\alpha_{3}$ are the roots of

$$
\begin{aligned}
& s^{3}+(3 a+3 b+2 c) s^{2}+\left(3 a^{2}+2 b^{2}+c^{2}+6 a b+2 b c+2 c a\right) s \\
& +a\left(a^{2}+3 a b+2 b^{2}\right)=0
\end{aligned}
$$

\subsubsection{Mean time before failure (MTBF)}

The system MTBF is the expected or average time to failure and is given by $M T B F=R^{*}(0)=$ $L_{0}(0)+L_{1}(0)+L_{2}(0)$ where $R^{*}(s)$ is the Laplace transform of $R(t)$ at $s$. Hence,

\subsubsection{System availability}

$$
M T B F=\frac{\left(3 a^{2}+2 b^{2}+c^{2}+6 a b+2 b c+2 c a\right)}{a\left(a^{2}+3 a b+2 b^{2}\right)}
$$

The system availability $A(t)$ is the probability that the system operates within the tolerances at a given instant of time $t$ and is obtained by solving for $p_{i}(t), i \in E$. The following system of differential-difference equations are obtained by using the infinitesimal generator given in Equation (2).

$$
\begin{aligned}
& \frac{d p_{0}(t)}{d t}=-(a+2 b) p_{0}(t)+c p_{1}(t) \\
& \frac{d p_{1}(t)}{d t}=(a+2 b) p_{0}(t)-(a+b+c) p_{1}(t)+c p_{2}(t) \\
& \frac{d p_{2}(t)}{d t}=(a+b) p_{1}(t)-(c+a) p_{2}(t)+c p_{3}(t)
\end{aligned}
$$




$$
\frac{d p_{3}(t)}{d t}=a p_{2}(t)-c p_{3}(t)
$$

Using Laplace transform on both sides of the above differential-difference equations and solving, the expressions for $p_{i}(t), i=0,1,2,3$ are obtained, respectively, as

$$
\begin{aligned}
& \frac{-c^{3}}{\alpha_{1} \alpha_{2} \alpha_{3}}+c(a+2 b) \sum_{i=1}^{3} \frac{\left[\alpha_{i}^{2}+(a+2 c) \alpha_{i}+c^{2}\right]}{\alpha_{i}\left(\alpha_{i}+a+2 b\right) \prod_{j=1, j \neq i}^{3}\left(\alpha_{i}-\alpha_{j}\right)} e^{\alpha_{i} t} \\
& \frac{-(a+2 b) c^{2}}{\alpha_{1} \alpha_{2} \alpha_{3}}+(a+2 b) \sum_{i=1}^{3} \frac{\left[\alpha_{i}^{2}+(a+2 c) \alpha_{i}+c^{2}\right]}{\alpha_{i} \prod_{j=1, j \neq i}^{3}\left(\alpha_{i}-\alpha_{j}\right)} e^{\alpha_{i} t} \\
& \frac{-(a+b)(a+2 b) c}{\alpha_{1} \alpha_{2} \alpha_{3}}+(a+b)(a+2 b) \sum_{i=1}^{3} \frac{\left(\alpha_{i}+c\right)}{\alpha_{i} \prod_{j=1, j \neq i}^{3}\left(\alpha_{i}-\alpha_{j}\right)} e^{\alpha_{i} t} \\
& \frac{-a(a+b)(a+2 b)}{\alpha_{1} \alpha_{2} \alpha_{3}}+a(a+b)(a+2 b) \sum_{i=1}^{3} \frac{1}{\alpha_{i} \prod_{j=1, j \neq i}^{3}\left(\alpha_{i}-\alpha_{j}\right)} e^{\alpha_{i} t}
\end{aligned}
$$

where $\alpha_{1}, \alpha_{2}$, and $\alpha_{3}$ are the roots of the equation

$$
\begin{aligned}
s^{3} & +3(a+b+c) s^{2}+\left[(a+2 c)(a+2 b+c)+(a+b)(2 a+2 b+c)+c^{2}\right] s+\left[c^{3}\right. \\
& \left.+(a+2 b)\left(a^{2}+c^{2}+a b+b c+c a\right)\right]=0 .
\end{aligned}
$$

Hence, the system availability is given by

$$
A(t)=p_{0}(t)+p_{1}(t)+p_{2}(t)
$$

\subsubsection{Steady state availability}

The system steady state availability is the expected fractional amount of time in a continuum of operating time that the system is in upstate and is given by

$$
\begin{aligned}
A_{\infty} & =\lim _{\mathrm{t} \rightarrow \infty} A(t) \\
& =\frac{\mathrm{c}\left[c^{2}+(\mathrm{a}+2 \mathrm{~b})(a+b+c)\right]}{\left[c^{3}+(\mathrm{a}+2 \mathrm{~b})\left(a^{2}+c^{2}+a b+b c+c a\right)\right]}
\end{aligned}
$$

\section{Particular case:}

The equations for system reliability, MTBF, system availability, and steady state availability when the lifetimes of online and standby units and the repair time of a failed unit are independent can be obtained by taking $\lambda_{4}=0$ in Equations (6), (7), (16), and (17), respectively.

\section{Confidence interval for steady state availability of system}

\subsection{Moment and CAN estimators for steady state availability of system}

Let $\left(Y_{1 i}, Y_{2 i}, Y_{3 i}, Y_{4 i}\right), i=1,2, \ldots, n$ be a random sample of size $n$ drawn from a quadrivariate exponential lifetimes and repair time population with the survival function given by Equation (1). It is clear that $\bar{Y}_{1}, \bar{Y}_{2}, \bar{Y}_{3}\left(=\bar{Y}_{2}\right)$ and $\bar{Y}_{4}$ are the moment estimators of $\frac{1}{\left(\lambda_{1}+\lambda_{4}\right)}, \frac{1}{\left(\lambda_{2}+\lambda_{4}\right)}, \frac{1}{\left(\lambda_{2}+\lambda_{4}\right)}$, and $\frac{1}{\left(\lambda_{3}+\lambda_{4}\right)}$, respectively, where $\bar{Y}_{1}, \bar{Y}_{2}, \bar{Y}_{3}\left(=\bar{Y}_{2}\right)$ and $\bar{Y}_{4}$ are the sample means of lifetimes of online and standby units and repair time of a failed unit, respectively.

Let $\theta_{i}=\frac{1}{\left(\lambda_{i}+\lambda_{4}\right)}, i=1,2,3$. Using $\theta_{i}, i=1,2,3$ in Equation (17) and substituting its corresponding moment estimators, the estimator of the steady state availability of the system $A_{\infty}$ 
based on moments is obtained as

$$
\hat{A}_{\infty}=\frac{\bar{Y}_{1}\left[\bar{Y}_{1}^{2} \bar{Y}_{2}^{2}+\bar{Y}_{4}\left(2 \bar{Y}_{1}+\bar{Y}_{2}\right)\left(\bar{Y}_{1} \bar{Y}_{2}+\bar{Y}_{2} \bar{Y}_{4}+\bar{Y}_{4} \bar{Y}_{1}\right)\right]}{\left[\bar{Y}_{1}^{3} \bar{Y}_{2}^{2}+\bar{Y}_{2}^{2} \bar{Y}_{4}^{3}+\bar{Y}_{4} \bar{Y}_{1}\left(\bar{Y}_{4}+\bar{Y}_{1}\right)\left(2 \bar{Y}_{4} \bar{Y}_{1}+\bar{Y}_{2}^{2}+3 \bar{Y}_{2} \bar{Y}_{4}\right)+2 \bar{Y}_{1}^{3} \bar{Y}_{2} \bar{Y}_{4}\right]}
$$

It may be noted that $\hat{A}_{\infty}$ given in Equation (18) is a real-valued function in $\bar{Y}_{1}, \bar{Y}_{2}$, and $\bar{Y}_{4}$, which is also differentiable. Consider the following multivariate central limit theorem (see Radhakrishna Rao, 1974).

\subsubsection{Multivariate central limit theorem}

Suppose $T_{1}{ }^{\prime}, T_{2}{ }^{\prime}, T_{3}{ }^{\prime}, \ldots$ are independent and identically distributed $k$-dimensional random variables such that $T_{n}{ }^{\prime}=\left(T_{1 n}, T_{2 n}, \ldots, T_{k n}\right), n=1,2,3, \ldots$ having the first and second order moments $E\left(T_{n}\right)=\mu$ and $\operatorname{var}\left(T_{n}\right)=\Sigma$, respectively. Define the sequence of random variables $\bar{T}_{n}^{\prime}=\left(\bar{T}_{1 n}, \bar{T}_{2 n}, \ldots, \bar{T}_{k n}\right), n=1,2,3, \ldots$ where $\bar{T}_{i n}=\frac{1}{n} \sum_{j=1}^{n} T_{i j}, i=1,2, \ldots, k$.

Then, $\sqrt{n}\left(\bar{T}_{n}-\mu\right) \stackrel{d}{\rightarrow} N_{k}(0$,$) as \mathrm{n} \rightarrow \infty$.

\subsubsection{CAN estimator}

By applying the multivariate central limit theorem given in Section 3.1.1, it is seen that $\sqrt{n}\left[\left(\bar{Y}_{1}, \bar{Y}_{2}, \bar{Y}_{2}, \bar{Y}_{4}\right)-\left(\theta_{1}, \theta_{2}, \theta_{2}, \theta_{3}\right)\right] \stackrel{d}{\rightarrow} N_{4}(0, \sigma)$ as $n \infty$, where the dispersion matrix $\Sigma=$ $\left(\left(\sigma_{i j}\right)\right)$ is given by



Thus, $\sqrt{n}\left(\hat{A}_{\infty}-A_{\infty}\right) N_{4}\left(0, \sigma^{2}(\theta)\right)$, where $\theta=\left(\theta_{1}, \theta_{2}, \theta_{2}, \theta_{3}\right)$ and

$$
\begin{aligned}
\sigma^{2}(\theta)= & {\left[\theta_{1}^{2}\left(\frac{\partial A_{\infty}}{\partial \theta_{1}}\right)^{2}+\frac{4 \theta_{2}^{2}}{\left(2-\lambda_{4} \theta_{2}\right)}\left(\frac{\partial A_{\infty}}{\partial \theta_{2}}\right)^{2}+\theta_{3}^{2}\left(\frac{\partial A_{\infty}}{\partial \theta_{3}}\right)^{2}\right]+4 \lambda_{4} \theta_{2}^{2} } \\
& \times\left[\frac{\theta_{1}^{2}}{\left(\theta_{1}+\theta_{2}-\lambda_{4} \theta_{1} \theta_{2}\right)}\left(\frac{\partial A_{\infty}}{\partial \theta_{1}}\right)\left(\frac{\partial A_{\infty}}{\partial \theta_{2}}\right)+\frac{\theta_{3}^{2}}{\left(\theta_{2}+\theta_{3}-\lambda_{4} \theta_{2} \theta_{3}\right)}\left(\frac{\partial A_{\infty}}{\partial \theta_{2}}\right)\left(\frac{\partial A_{\infty}}{\partial \theta_{3}}\right)\right] \\
& +2 \frac{\lambda_{4} \theta_{1}^{2} \theta_{3}^{2}}{\left(\theta_{1}+\theta_{3}-\lambda_{4} \theta_{1} \theta_{3}\right)}\left(\frac{\partial A_{\infty}}{\partial \theta_{1}}\right)\left(\frac{\partial A_{\infty}}{\partial \theta_{3}}\right)
\end{aligned}
$$

By substituting for the partial derivatives $\left(\frac{\partial A_{\infty}}{\partial \theta_{i}}\right), i=1,2,3$ in Equation (20), we get an expression for $\sigma^{2}(\theta)$. Thus $\hat{A}_{\infty}$ is a CAN estimator of $A_{\infty}$. 


\subsection{Confidence interval for steady state availability of system}

Let $\sigma^{2}(\hat{\theta})$ be an estimator of $\sigma^{2}(\theta)$ obtained by replacing $\theta$ by a consistent estimator $\hat{\theta}$, namely $\hat{\theta}=\bar{Y}_{1}, \bar{Y}_{2}, \bar{Y}_{2}, \bar{Y}_{4}$. Let $\hat{\sigma}^{2}=\sigma^{2}(\hat{\theta})$. Since $\sigma^{2}(\theta)$ is a continuous function of $\theta, \hat{\sigma}^{2}$ is a consistent estimator of $\sigma^{2}(\theta)$. That is, $\hat{\sigma}^{2} \stackrel{P}{\rightarrow} \sigma^{2}(\theta)$ as $n \rightarrow \infty$. By Slutsky theorem, we have

$$
\frac{\sqrt{n}\left(\hat{A}_{\infty}-A_{\infty}\right)}{\hat{\sigma}} \stackrel{d}{\rightarrow} N(0,1)
$$

that is,

$$
\operatorname{Pr}\left(-k_{\frac{\alpha}{2}}<\frac{\sqrt{n}\left(\hat{A}_{\infty}-A_{\infty}\right)}{\hat{\sigma}}<k_{\frac{\alpha}{2}}\right)=(1-\alpha),
$$

where $k_{\frac{\alpha}{2}}$ is obtained from normal tables. Hence, a $100(1-\alpha) \%$ confidence interval for $\mathrm{A}_{\infty}$ is given by $\hat{A}_{\infty} \pm k_{\frac{\alpha}{2}} \frac{\hat{\sigma}}{\sqrt{n}}$, where $\hat{\sigma}$ is obtained from Equation (20).

\subsection{Estimator of system reliability based on moments}

We have already seen that $\bar{Y}_{1}, \bar{Y}_{2}, \bar{Y}_{2}$, and $\bar{Y}_{4}$ are the moment estimators of $\frac{1}{\left(\lambda_{1}+\lambda_{4}\right)}, \frac{1}{\left(\lambda_{2}+\lambda_{4}\right)}, \frac{1}{\left(\lambda_{2}+\lambda_{4}\right)}$, and $\frac{1}{\left(\lambda_{3}+\lambda_{4}\right)}$, respectively, where $\bar{Y}_{1}, \bar{Y}_{2}, \bar{Y}_{2}$, and $\bar{Y}_{4}$ are the sample means of lifetime of online unit, lifetimes of standby units, and repair time of a failed unit, respectively. Hence an estimator of system reliability can be obtained by using the moment estimators in Equation (6) and is given by

$$
\hat{R}(t)=\hat{p}_{0}(t)+\hat{p}_{1}(t)+\hat{p}_{2}(t)
$$

where

$$
\begin{aligned}
& \hat{p}_{0}(t)=\frac{1}{\bar{Y}_{1}^{2} \bar{Y}_{2} \bar{Y}_{4}^{2}} \sum_{i=1}^{3} \\
& \times \frac{\left[\hat{\alpha}_{i}^{2}\left(\bar{Y}_{1}^{2} \bar{Y}_{2} \bar{Y}_{4}^{2}\right)+\bar{Y}_{1} \bar{Y}_{4}\left(2 \bar{Y}_{4} \bar{Y}_{2}+\bar{Y}_{4} \bar{Y}_{1}+2 \bar{Y}_{1} \bar{Y}_{2}\right)+\left(\bar{Y}_{1} \bar{Y}_{4}^{2}+\bar{Y}_{1}^{2} \bar{Y}_{2}+\bar{Y}_{2} \bar{Y}_{4}^{2}+\bar{Y}_{1} \bar{Y}_{2} \bar{Y}_{4}\right)\right]}{\prod_{j=1, j \neq i}^{3}\left(\hat{\alpha}_{i}-\hat{\alpha}_{j}\right)} e^{\hat{\alpha}_{i} t} \\
& \hat{p}_{1}(t)=\frac{\left(2 \bar{Y}_{1}+\bar{Y}_{2}\right)}{\left(\bar{Y}_{1}^{2} \bar{Y}_{2} \bar{Y}_{4}\right)} \sum_{i=1}^{3} \frac{\left[\hat{\alpha}_{i} \bar{Y}_{1} \bar{Y}_{4}+\bar{Y}_{4}+\bar{Y}_{1}\right]}{\prod_{j=1, j \neq i}^{3}\left(\hat{\alpha}_{i}-\hat{\alpha}_{j}\right)} e^{\hat{\alpha}_{i} t} \\
& \hat{p}_{2}(t)=\frac{\left(\bar{Y}_{1}+\bar{Y}_{2}\right)\left(2 \bar{Y}_{1}+\bar{Y}_{2}\right)}{\left(\bar{Y}_{1} \bar{Y}_{2}\right)^{2}} \sum_{i=1}^{3} \frac{1}{\prod_{j=1, j \neq i}^{3}\left(\hat{\alpha}_{i}-\hat{\alpha}_{j}\right)} e^{\hat{\alpha}_{i} t}
\end{aligned}
$$

where $\hat{\alpha}_{1}, \hat{\alpha}_{2}$, and $\hat{\alpha}_{3}$ are the roots of

$$
\begin{aligned}
& \bar{Y}_{1}^{3} \bar{Y}_{2}^{2} \bar{Y}_{4}^{2} s^{3}+\bar{Y}_{1}^{2} \bar{Y}_{2} \bar{Y}_{4}\left(2 \bar{Y}_{1} \bar{Y}_{2}+3 \bar{Y}_{1} \bar{Y}_{4}+3 \bar{Y}_{2} \bar{Y}_{4}\right) s^{2} \\
& \quad+\bar{Y}_{1}\left(\bar{Y}_{1}^{2} \bar{Y}_{2}^{2}+3 \bar{Y}_{2}^{2} \bar{Y}_{4}^{2}+2 \bar{Y}_{1}^{2} \bar{Y}_{4}^{2}+6 \bar{Y}_{1} \bar{Y}_{2} \bar{Y}_{4}^{2}+2 \bar{Y}_{1}^{2} \bar{Y}_{2} \bar{Y}_{4}+2 \bar{Y}_{1} \bar{Y}_{2}^{2} \bar{Y}_{4}\right) s \\
& \quad+\bar{Y}_{4}^{2}\left(2 \bar{Y}_{1}^{2}+3 \bar{Y}_{1} \bar{Y}_{2}+\bar{Y}_{2}^{2}\right)=0 .
\end{aligned}
$$

\section{Numerical illustration}

In this section, numerical illustration of the behavior of the reliability of the system is provided by generating random samples of size $n=10,000$ each from the quadrivariate exponential 


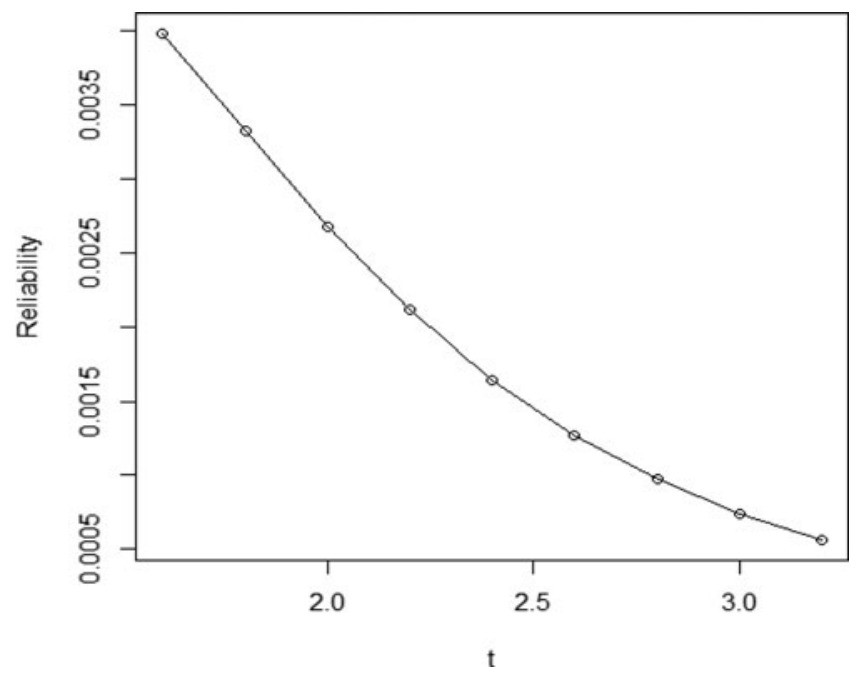

Figure 2. Line plot of the estimated values of the system reliability based on moments.

distribution using $\mathrm{R}$ language, version 3.0.2, by fixing the values of the various parameters as $\lambda_{1}=2, \lambda_{2}=3, \lambda_{3}=3$, and $\lambda_{4}=1$, respectively. The estimated values of the system reliability $(\hat{R}(t))$ based on moments given in Equation $(21)$ is evaluated for various choices of time periods $t=1.6,1.4, \ldots, 3.2$. The following are the values of $\hat{R}(t)$ obtained for various choices of $t$.

\begin{tabular}{cccccccccc}
\hline$t$ & 1.6 & 1.8 & 2.0 & 2.2 & 2.4 & 2.6 & 2.8 & 3.0 & 3.2 \\
$\hat{R}(t)$ & 0.00398 & 0.00332 & 0.00267 & 0.00211 & 0.00164 & 0.00126 & 0.00097 & 0.00074 & 0.00056 \\
\hline
\end{tabular}

The line plot of $(t, \hat{R}(t))$ is shown Figure 2 .

It is evident from the plot that as $t$ increases, the value of $\hat{R}(t)$ decreases agreeing with the theoretical results.

\section{Conclusion}

In this paper, a three-unit warm standby system with dependent structure, wherein the lifetimes of online unit, standby units, and the repair time of failed units is governed by quadrivariate exponential law is studied. Measures of system performance such as reliability, MTBF, availability, and steady state availability are obtained. Further, a $100(1-\alpha) \%$ confidence interval for the steady state availability of the system and an estimator of system reliability based on moments are obtained. Numerical work is carried out to illustrate the behavior of the system reliability based on moments by simulating samples from quadrivariate exponential distribution. Generalization of the above results to a $n(\geq 4)$ unit warm standby system with $r(\geq 2)$ repair facilities is being investigated.

\section{Acknowledgments}

The authors thank the referees for their useful comments and suggestions, which have helped in improving the presentation of the paper. 


\section{References}

Barlow, R.E. and Proschan, F. (1975). Statistical Theory of Reliability and Life Testing. New York: HoltReinhart.

Chandrasekhar, P., Natarajan, R. (1994). Confidence limits for steady state availability of a two unit standby system. Microelectron. Reliab. 34(7):1249-1251.

Chandrasekhar, P., Vaidyanathan, V.S., Yadavalli, V.S.S., Xavier, S. (2013). Statistical inference for a two unit standby system with dependent structure. OPSEARCH 50(3):372-382.

Marshall, A.W., Olkin, I. (1967). A Multivariate exponential distribution. J. Amer. Statist. Assoc. 62: $30-44$.

Osaki, S., Nakagawa, T. (1976). Bibliography for reliability and availability of stochastic systems. IEEE Trans. on Reliab. R-25:284-287.

Radhakrishna Rao, C. (1974). Linear statistical inference and its applications. New Delhi: Wiley Eastern Pvt. Ltd.

Srinivasan, S.K., Subramanian, R. (2006). Reliability analysis of a three unit warm standby redundant system with repair. Ann. Oper. Res. 143:227-235. 